\title{
Maze preferences in naive rats produced by injection of ribonucleic acid from trained rats'
}

\author{
ALLAN L. JACOBSON, FRANK R. BABICH, SUZANNE BUBASH ${ }^{2}$ AND CAROLYN GOREN \\ DEPARTMENT OF PSYCHOLOGY, UNIVERSITY OF CALIFORNIA, LOS ANGELES
}

One group of rats was trained with food reward to choose alternative $A$ in a two-choice discrimination apparatus, and a second group was trained to choose alternative B. Ribonucleic acid was extracted from the brains of the se trained rats and was injected into untrained rats. On subsequent unreinforced test trials the untrained rats showed a significant tendency to choose the alternative to which their respective donor rats had been trained.

In two recent experiments (Babich, Jacobson, Bubash, \& Jacobson, 1965; Jacobson, Babich, Bubash, \& Jacobson, 1965), we found that when ribonucleic acid (RNA) from the brains of trained rats was injected into untrained rats, the latter subsequently performed the originally-trained response. The learned behavior involved in these experiments consisted of approaching the food cup in a Skinner box when a distinct stimulus (click or blinking light) was presented. Our results indicated that the response transfer was specific in nature and did not occur in any of several appropriate control groups.

One of the questions raised by these experiments is that of whether the response transfer might be demonstrated in a different behavioral situation. In the present experiment, the RNA transfer effect is examined in a two-choice discrimination apparatus. As in our previous experiments, the injected animals were never rewarded during testing, but were simply given the opportunity in a series of unreinforced test trials to perform the response learned by the donor animals. Subjects and Apparatus

Ss were 90-110-day-old female Sprague-Dawley rats weighing approximately $250 \mathrm{gm}$.

The discrimination apparatus is shown in Fig. 1.

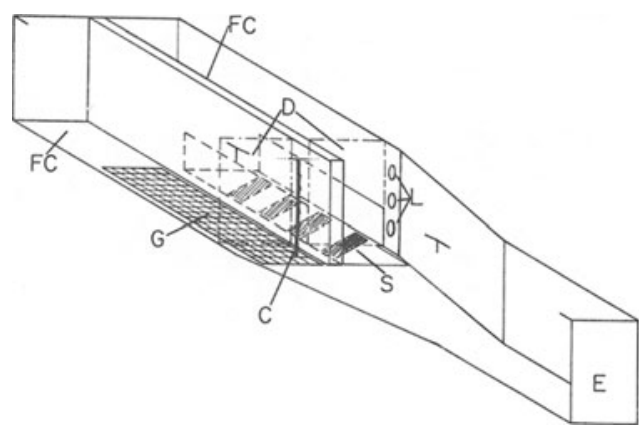

Fig. 1. The discrimination apparatus. Key: $F C=$ food $\operatorname{cup} ; \mathrm{D}=$ guillotine door; $G=$ grid floor; $C=$ beaded chain; $S=$ stripe; $L=$ jewel lights; $E=$ entrance.
A guillotine door was located at the entrance to each alley. Once the rat's entire body (except the tail) was past the entrance, the door to that alley was closed. As can be seen in Fig. 1, the two alternatives differed in a number of respects. One alternative (A) had a fine mesh screen on the floor and a beaded chain hanging in the alley entrance. The other alternative (B) had white contact paper with black tape stripes on the floor and a vertical column of white jewel lights to one side of the alley entrance. Each jewel light was illuminated by a small bulb. Preliminary work showed that with cues combined in this particular way, rats tended to choose A and B with roughly equal frequency. Pellets could be dropped into the food dishes at the ends of the alleys via polyethylene tubes to which funnels were attached. Overhead lighting was diffused, and no attempt was made to reduce extra-maze stimuli.

\section{Procedure}

Initially, each of eight rats was adapted to the maze for $15 \mathrm{~min}$. and on the following day was given 11 unreinforced "preference" trials. On preference trials, the rat was placed in the stem and was permitted to run to one side. The door was then dropped and the rat was confined for $15 \mathrm{sec}$. before being removed from the maze. This procedure also defined a trial during training and during post-injection testing. Preference trials for the first eight rats showed that alternatives $\mathrm{A}$ and $\mathrm{B}$ were chosen equally ( 44 choices of each) and that individual biases tended to be small (no rat more extreme than 7 out of 11 choices of one side). On this basis, we decided not to preferencetest the rats which were to be injected. Five of the eight rats were then trained in the maze to choose the side they had selected less often on preference trials. For each correct choice the rat was rewarded with four 45-mg Noyes pellets. After each incorrect choice, the rat was simply confined in the alley for $15 \mathrm{sec}$. Each rat was run on a given day until it had made 25 correct choices and therefore received 100 pellets. No other food was given to these rats during training. Each rat was trained for five consecutive days. By the fifth day, none of the 42 rats trained in the experiment made more than two incorrect choices per daily 25 correct choices.

On the day of completion of training, each of the five rats was sacrificed with ether, and the brain was taken out as quickly as possible. A cut was made on a line joining the superior colliculus to the rostral end of the pons. The tissue posterior to this cut was discarded, as was the tissue of the olfactory bulbs. The 
average weight of the tissue retained was $1.3 \mathrm{gm}$. RNA was then extracted from this tissue by a phenol extraction technique (for details of the procedure, see Babich et al, 1965). The average yield was $1.0 \mathrm{mg} / 1.0$ $\mathrm{gm}$ of tissue. The RNA extracted from each brain was dissolved in $2.0 \mathrm{ml}$ of isotonic saline.

Approximately $8 \mathrm{hr}$. after extraction, the RNA from each of the trained rats was injected intraperitoneally with a 3/4-in 22-gauge needle into an untrained test rat (the xiphoid process was used as a guide for the injection). Prior to injection, each of the test rats had been adapted to the maze for three days, $15 \mathrm{~min}$. per day. The test animals were assigned code letters, and from this point on, all testing was conducted "blind": the testers did not know the group membership of any animal until the completion of testing.

A session of testing for a given rat consisted of permitting that rat to make five runs in the maze. On each trial, the animal was confined for $15 \mathrm{sec}$. in the side it chose and was then returned to its home cage. The intertrial interval was 4-6 min. Five such testing sessions were given, at $6,9,12,22$, and $25 \mathrm{hr}$. after injection. Each test animal thus received a total of 25 trials. No food was ever given in the maze during testing. At the beginning of testing, all rats were approximately $24 \mathrm{hr}$. food-deprived. After the third test session, all rats were fed 4-5 gm of Purina Lab Chow.

The five recipient rats from the first week were subsequently trained in the maze (to the side of their respective donor animals) and served as RNA donors in the second week of the experiment. This process was repeated for the remaining four weeks of the experiment as well. In addition, three new rats were trained and used as donors during the third week. Thus, in all, 42 rats were trained in the maze (22 to $A, 20$ to $B$ ) and served as RNA donors, and 42 recipient rats were tested.

\section{Resulfs and Discussion}

Table 1 shows the "preferences" of the test animals as indexed by their predominant choices on the 25 test trials, regardless of the strength of preference. As the table shows, rats injected with RNA from A-trained donors tended to choose $A$ on test trials, and "B-injected" rats tended to choose B. By a $\chi^{2}$ test, the differences are significant at the .02 level (onetailed test). Absolute preferences were on the whole small (see Table 2).
Table 1. Preferences of injected rats on the 25 test trials. Cell entries indicate the number of rats choosing that alternative.

\begin{tabular}{|c|c|c|c|}
\hline & & \multicolumn{2}{|c|}{ Preference of injected rats } \\
\hline $\begin{array}{l}\text { Side to which } \\
\text { donor was trained }\end{array}$ & $\begin{array}{l}\mathrm{A} \\
\mathrm{B}\end{array}$ & $\begin{array}{r}15 \\
6\end{array}$ & $\begin{array}{r}7 \\
14\end{array}$ \\
\hline
\end{tabular}

Table 2. Distribution of scores for recipient rats. Each animal's score is the number of times (out of 25) it selected the alternative ( $A$ or $B$ ) to which its respective donor rat had been trained.

\begin{tabular}{cc} 
Seore & Frequency \\
\hline 7 & 1 \\
9 & 2 \\
10 & 3 \\
11 & 2 \\
12 & 5 \\
13 & 8 \\
14 & 7 \\
15 & 10 \\
16 & 1 \\
17 & 1 \\
19 & 1 \\
21 & 1 \\
\hline
\end{tabular}

Apparently, then, the specific training given to donor rats was a determinant of the subsequent choices of recipient rats. In addition to confirming our earlier findings in the Skinner box, the present results further indicate that the RNA transfer effect is quite specific in nature. Moreover, the fact that this effect can be demonstrated in widely disparate behavioral situations provides support for the notion that RNA may be critically involved in many memory storage phenomena.

\section{References}

Babich, F. R., Jacobson, A. L., Bubach, S., \& Jacobson, Ann. Transfer of a response to naive rats by injection of ribonucleic acid extracted from trained rats. Science, 1965, 149, 656-657. Jacobson, A. L., Babich, F. R., Bubash, S., \& Jacobson, Ann. Differential approach tendencies produced by injection of ribonucleic acid from trained rats. Science, 1965, 150, 636-637. Notes

1. This research was supported by NSF Research Grant GB-3524 and University of California Research Grant 2068 to the senior author.

2. Now at Lawrence Radiation Laboratory, Livermore, California. 